\title{
Time-lapse Systems in Assisted Reproduction: The Importance of the Earliest Steps of Fertilization
}

\author{
Bernd Rosenbusch \\ Department of Gynaecology and Obstetrics, University of Ulm, Germany
}

\section{LETTER}

Dear Editor-in-Chief:

The Editorial of this year's second issue of your interesting journal commented on the increasing use of time-lapse ( $T L$ ) image systems in laboratories of assisted reproduction (Franco Jr, 2015). It was concluded that possible advantages of TL systems over classical morphological analyses are still lacking clear evidence. Moreover, new parameters assessing the pronuclear stage obviously have been introduced quite recently whereas the classical TL parameters mostly focused on the kinetics of embryo development (Franco Jr, 2015). Particularly with respect to deviations from the normal number of pronuclei (PN) I should like to add some thoughts.

First, the formation of a supernumerary pronucleus is relatively common. However, whereas three (or even more) PN of approximately the same size may easily be recognized, there are cases with two normal-sized PN that are accompanied by an additional, rather small pronucleus. The basic mechanisms responsible for this phenomenon have been proposed earlier: either an incomplete chromatid segregation into the second polar body (PB) or an dispersal of the oocyte chromatids in the presence of a normal second PB (Rosenbusch, 2010). Because such abnormalities lead to irregular chromosome distributions into the blastomeres, pronuclear stages are carefully checked at 200x magnification in our laboratory and affected oocytes are excluded from transfer or cryopreservation. It has been noted that parameters associated with pronuclear morphology and dynamics may not be clearly observable with a dark-field TL system and that multiple planes will be necessary if the oocyte is not rotated (Ciray et al., 2014). Accordingly, the appropriate system has to be selected with great care if a precise inspection of the pronuclear constitution is envisaged. Of course, one could think of giving up the early PN assessment in favour of detecting aneuploidy at later embryonic stages since there are indications that aneuploid and normal embryos differ in the initiation of compaction and blastulation (Campbell et al., 2013) or, more generally, in their kinetic behaviour (Basile et al., 2014). On the other hand, it is questionable whether the latter approach that involves discarding (presumably aneuploid) cleavage stages will be an option in countries with strict embryo protection laws.

Second, the appearance of a single pronucleus is another undesired event IVF laboratories have to deal with. As recently discussed (Rosenbusch, 2014), some embryos obtained from monopronuclear oocytes are biparental diploid and might be considered for transfer. How can the unexpected diploid constitution be explained? A single diploid pronucleus could arise when the paternal and maternal genomes are immediately enclosed in a common pronuclear envelope. Alternatively, two individual PN could be formed first but quickly undergo membrane fusion resulting in a so-called 'syngamy nucleus'. In contrast, Azevedo et al. (2014) proposed premature pronuclear envelope breakdown (pPNBD) as another mechanism in single pronucleated zygotes. Because of a low rate of normal diploidy in affected zygotes, the latter authors do not recommend to use the resulting embryos for transfer. These brief considerations show that continuous monitoring of many oocytes during the period between sperm penetration and appearance of PN is necessary to obtain more information on such phenomena, for instance to ascertain whether early pronuclear membrane fusion exists and to assess the frequency of pPNBD.

In short, time-lapse monitoring represents a promising technique that hopefully will be developed further to satisfy all specific laboratory requirements and that may help to address some yet unresolved scientific issues.

\section{CONFLICT OF INTERESTS}

No conflict of interest have been declared.

\section{Corresponding author:}

Bernd Rosenbusch

Department of Gynaecology and Obstetrics

University of Ulm - Prittwitzstrasse 43

89075 Ulm, Germany

E-mail: bernd.rosenbusch@uniklinik-ulm.de

\section{REFERENCES}

Azevedo AR, Pinho MJ, Silva J, Sá R, Thorsteinsdóttir S, Barros A, Sousa M. Molecular cytogenetics of human single pronucleated zygotes. Reprod Sci. 2014;21:1472-82.

Basile N, Nogales $M$ delC, Bronet F, Florensa M, Riqueiros M, Rodrigo L, Garcia-Velasco J, Meseguer M. Increasing the probability of selecting chromosomally normal embryos by time-lapse morphokinetics analysis. Fertil Steril. 2014;101:699-704.

Campbell A, Fishel S, Bowman N, Duffy S, Sedler M, Hickman CFL. Modelling a risk classification of aneuploidy in human embryos using non-invasive morphokinetics. Reprod Biomed Online. 2013;26:477-85.

Ciray HN, Campbell A, Agerholm IE, Aguilar J, Chamayou S, Esbert M, Sayed S. Proposed guidelines on the nomenclature and annotation of dynamic human embryo monitoring by a time-lapse user group. Hum Reprod. 2014;29:265060.

Franco JG Jr. The world of embryo morphokinetics of timelapse systems. JBRA Assist. Reprod. 2015;19:36.

Rosenbusch B. A preliminary concept, deduced from cytogenetic analyses, for explaining different types of multipronuclear oocytes obtained after ICSI. Fertil. Steril. 2010;94:2479-81.

Rosenbusch B. The chromosomal constitution of embryos arising from monopronuclear oocytes in programmes of assisted reproduction. Int. J. Reprod. Med. 2014; 2014:418198 